\title{
Research Article \\ On Output Feedback Multiobjective Control for Singularly Perturbed Systems
}

\section{Mehdi Ghasem Moghadam and Mohammad Taghi Hamidi Beheshti}

\author{
Control and Communication Networks Laboratory, Electrical Engineering Department, \\ Tarbiat Modares University, Tehran, Iran \\ Correspondence should be addressed to \\ Mohammad Taghi Hamidi Beheshti, mbehesht@modares.ac.ir
}

Received 6 September 2010; Revised 30 November 2010; Accepted 6 January 2011

Academic Editor: Alexei Mailybaev

Copyright (C) 2011 M. Ghasem Moghadam and M. T. H. Beheshti. This is an open access article distributed under the Creative Commons Attribution License, which permits unrestricted use, distribution, and reproduction in any medium, provided the original work is properly cited.

\begin{abstract}
A new design procedure for a robust $H_{2}$ and $H_{\infty}$ control of continuous-time singularly perturbed systems via dynamic output feedback is presented. By formulating all objectives in terms of a common Lyapunov function, the controller will be designed through solving a set of inequalities. Therefore, a dynamic output feedback controller is developed such that $H_{\infty}$ and $H_{2}$ performance of the resulting closed-loop system is less than or equal to some prescribed value. Also, $H_{\infty}$ and $H_{2}$ performance for a given upperbound of singular perturbation parameter $\varepsilon \in\left(0, \varepsilon^{*}\right]$ are guaranteed. It is shown that the $\varepsilon$-dependent controller is well defined for any $\varepsilon \in\left(0, \varepsilon^{*}\right]$ and can be reduced to an $\varepsilon$-independent one so long as $\varepsilon$ is sufficiently small. Finally, numerical simulations are provided to validate the proposed controller. Numerical simulations coincide with the theoretical analysis.
\end{abstract}

\section{Introduction}

It is well known that the multiple time-scale systems, otherwise known as singularly perturbed systems, often raise serious numerical problems in the control engineering field. In the past three decades, singularly perturbed systems have been intensively studied by many researchers [1-8].

In practice, many systems involve dynamics operating on two or more time-scales [3]. In this case, standard control techniques lead to ill-conditioning problems. Singular perturbation methods can be used to avoid such numerical problems [1]. By utilizing the time scale properties, the system is decomposed into several reduced order subsystems. Reduced order controllers are designed for each subsystems.

In the framework of singularly perturbed systems, $H_{\infty}$ control has been investigated by many researchers, and various approaches have been proposed in this field [9-14]. 
However, to the best of our knowledge, the problem of multiobjective control for linear singular perturbed systems is still an open problem. By multiobjective control, we refer to synthesis problems with a mix of time- and frequency-domain specifications ranging from $H_{2}$ and $H_{\infty}$ performances to regional pole placement, asymptotic tracking or regulation, and settling time or saturation constraints.

In [12], the $H_{\infty}$ control problem is concerned via state feedback for fast sampling discrete-time singularly perturbed systems. A new $H_{\infty}$ controller design method is given in terms of solutions to linear matrix inequalities (LMIs), which eliminate the regularity restrictions attached to the Riccati-based solution. In [13], the $H_{\infty}$ control problem via state feedback for fast sampling discrete-time singularly perturbed systems is investigated. In fact, a new sufficient condition which ensures the existence of state feedback controllers is presented such that the resulting closed-loop system is asymptotically stable. In addition, the results were extended to robust controller design for fast sampling discrete-time singularly perturbed systems with polytopic uncertainties. Presented condition, in terms of a linear matrix inequality (LMI), is independent of the singular perturbation parameter.

Undoubtedly, output feedback stabilization is one of the most important problems in control theory and applications. In many real systems, the state vector is not always accessible and only partial information is available via measured output. Furthermore, the reliability of systems and the simplicity of implementation are other reasons of interest in output control which is adopted to stabilize a system.

LMI's have emerged as a powerful formulation and design technique for a variety of linear control problems. Since solving LMI's is a convex optimization problem, such formulations offer a numerically tractable means of attacking problems that lack an analytical solution. Consequently, reducing a control design problem to an LMI can be considered as a practical solution to this. Since the nonconvex formulation of the output feedback control, its conditions are restrictive or not numerically tractable [15]. It has been an open question how to make these conditions tractable by means of the existing software. Many research results on such a question have been reported in [15-20].

In $[1,2]$, the dynamic output feedback control of singular perturbation systems has been investigated. However, to the best of our knowledge, the design of dynamic output feedback for robust controller via LMI optimization is still an open problem. The main contribution of this paper is to solve the problem of multiobjective control for linear singularly perturbed systems. Considered problem consists of $H_{\infty}$ control, $H_{2}$ performance, and singular perturbation bound design. For given an $H_{\infty}$ performance bound $\gamma$ or $\mathrm{H}_{2}$ performance bound $v$, and an upperbound $\varepsilon^{*}$ for the singular perturbation, an $\varepsilon$-dependent dynamic output feedback controller will be constructed, such that for all $\varepsilon \in\left(0, \varepsilon^{*}\right]$. Due to this, the closed-loop system is admissible and the $H_{\infty}$ norm $\left(H_{2}\right.$ norm) of the closed-loop system is less than a prescribed $\gamma$ (prescribed $v$ ). Sufficient conditions for such a controller are obtained in form of strict LMIs. A mixed control $\mathrm{H}_{2} / \mathrm{H}_{\infty}$ problem for singular systems is also considered in this paper. It is shown that the designed controller is well-defined for for all $\varepsilon \in\left(0, \varepsilon^{*}\right]$. It is shown that if $\varepsilon$ is sufficiently small, the controller can be reduced to an $\varepsilon$-independent one. Numerical examples are given to illustrate the main results.

The paper is organized as follows. Section 2 gives the problem statement and motivations. Section 3 presents the main results. The theorems for $H_{2}, H_{\infty}$ and multiobjective $\mathrm{H}_{2} / \mathrm{H}_{\infty}$ design via output feedback control are presented in this section. Due to proposed theorems, robust $\mathrm{H}_{2}, \mathrm{H}_{\infty}$, and multiobjective performance of continuous-time singularly perturbed systems via dynamic output feedback for a linear systems will be accessible. 
Section 4 illustrates numerical simulations for the proposed theorems. Finally, conclusions in Section 5 close the paper.

Notation. A star $(*)$ in a matrix indicates a transpose quantity. For example: $(*)+A>0$ stands for $A^{T}+A>0$, or in a symmetric matrix $\left[\begin{array}{ll}A & * \\ B & C\end{array}\right]$ stands for $\left[\begin{array}{cc}A & B^{T} \\ B & C\end{array}\right]$.

\section{Problem Statement}

Consider the following singularly perturbed system with slow and fast dynamics described in the standard "singularly perturbed" form:

$$
\begin{gathered}
\dot{x}_{1}=A_{1} x_{1}+A_{2} x_{2}+B_{1} u+B_{w 1} w, \\
\varepsilon \dot{x}_{2}=A_{3} x_{1}+A_{4} x_{2}+B_{2} u+B_{w 2} w, \\
z_{1}=C_{z 11} x_{1}+C_{z 12} x_{2}+D_{z u 1} u+D_{z w 1} w, \\
z_{2}=C_{z 21} x_{1}+C_{z 22} x_{2}+D_{z u 2} u, \\
y=C_{y 1} x_{1}+C_{y 2} x_{2}+D_{y w} w,
\end{gathered}
$$

where $x_{1}(t) \in \mathfrak{R}^{n_{1}}$ and $x_{2}(t) \in \mathfrak{R}^{n_{2}}$ form the state vector, $u(t) \in \mathfrak{R}^{p}$ is the control input vector, $y(t) \in \mathfrak{R}^{m_{1}}$ is the output, $w(t)$ is a vector of exogenous inputs (such as reference signals, disturbance signals, sensor noise) and $z_{1}, z_{2}$ are regulated outputs.

By introducing the following notations:

$$
\begin{gathered}
x=\left[\begin{array}{l}
x_{1} \\
x_{2}
\end{array}\right], \quad A=\left[\begin{array}{ll}
A_{1} & A_{2} \\
A_{3} & A_{4}
\end{array}\right], \\
B=\left[\begin{array}{l}
B_{1} \\
B_{2}
\end{array}\right], \quad C_{y}=\left[\begin{array}{ll}
C_{y 1} & C_{y 2}
\end{array}\right], \\
C_{z 1}=\left[\begin{array}{ll}
C_{z 11} & C_{z 12}
\end{array}\right], \quad C_{z 2}=\left[\begin{array}{ll}
C_{z 21} & C_{z 22}
\end{array}\right], \\
B_{w}=\left[\begin{array}{l}
B_{w 1} \\
B_{w 2}
\end{array}\right] .
\end{gathered}
$$

The system (2.1) can be rewritten into the following compact form:

$$
\begin{gathered}
E_{\varepsilon} \dot{x}=A x+B u+B_{w} w, \\
z_{1}=C_{z 1} x+D_{z u 1} u+D_{z w 1} w, \\
z_{2}=C_{z 2} x+D_{z u 2} u, \\
y=C_{y} x+D_{y w} w .
\end{gathered}
$$


By applying dynamic output feedback controller in the following form:

$$
\begin{gathered}
\dot{x}_{c 1}=A_{c 1} x_{c 1}+A_{c 2} x_{c 2}+B_{c 1} y, \\
\varepsilon \dot{x}_{c 2}=A_{c 3} x_{c 1}+A_{c 4} x_{c 2}+B_{c 2} y, \\
u=\left[\begin{array}{ll}
C_{c 1} & C_{c 2}
\end{array}\right]\left[\begin{array}{l}
x_{c 1} \\
x_{c 2}
\end{array}\right]+D_{c} y .
\end{gathered}
$$

The controller (2.4) can be rewritten into the following compact form:

$$
\begin{gathered}
E_{\varepsilon} \dot{x}_{c}=A_{c} x_{c}+B_{c} y, \\
u=C_{c} x_{c}+D_{c} y,
\end{gathered}
$$

where

$$
A_{c}=\left[\begin{array}{ll}
A_{c 1} & A_{c 2} \\
A_{c 3} & A_{c 4}
\end{array}\right], \quad B_{c}=\left[\begin{array}{l}
B_{c 1} \\
B_{c 2}
\end{array}\right], \quad C_{c}=\left[\begin{array}{ll}
C_{c 1} & C_{c 2}
\end{array}\right]
$$

The closed loop system is

$$
\begin{aligned}
& {\left[\begin{array}{cc}
E_{\varepsilon} & 0 \\
0 & I
\end{array}\right]\left[\begin{array}{c}
\dot{x} \\
\dot{x}_{c}
\end{array}\right]=\left[\begin{array}{cc}
A+B D_{c} C_{y} & B C_{c} \\
E_{\varepsilon}^{-1} B_{c} C_{y} & E_{\varepsilon}^{-1} A_{c}
\end{array}\right]\left[\begin{array}{c}
x \\
x_{c}
\end{array}\right]+\left[\begin{array}{c}
B_{w}+B D_{c} D_{y w} \\
E_{\varepsilon}^{-1} B_{c} D_{y w}
\end{array}\right] w(t),} \\
& z_{i}=\left[\begin{array}{ll}
C_{z i}+D_{z u i} D_{c} C_{y} & D_{z u i} C_{c}
\end{array}\right]\left[\begin{array}{l}
x \\
x_{c}
\end{array}\right]+\left(D_{z w i}+D_{z u i} D_{c} D_{y w}\right) w(t), \quad \text { for } i=1,2 \text {, }
\end{aligned}
$$

where $D_{\mathrm{cl} 2}=0$ and

$$
\begin{gathered}
E_{e}=\operatorname{diag}\left(E_{\varepsilon}, I\right), \quad E_{\varepsilon}=\operatorname{diag}(I, \varepsilon I), \quad x_{\mathrm{cl}}=\left[\begin{array}{ll}
x^{T} & x_{c}^{T}
\end{array}\right]^{T}, \\
A_{\mathrm{cl}}=\left[\begin{array}{cc}
A+B D_{c} C_{y} & B_{w} C_{c} \\
E_{\varepsilon}^{-1} B_{c} C_{y} & E_{\varepsilon}^{-1} A_{c}
\end{array}\right], \\
B_{\mathrm{cl}}=\left[\begin{array}{c}
B_{w}+B D_{c} D_{y w} \\
E_{\varepsilon}^{-1} B_{c} D_{y w}
\end{array}\right], \\
C_{\mathrm{cl} i}=\left[\begin{array}{ll}
C_{z i}+D_{z u i} D_{c} C_{y} & D_{z u i} C_{c}
\end{array}\right], \\
D_{\mathrm{cl} 1}=\left(D_{z w 1}+D_{z u 1} D_{c} D_{y w}\right) .
\end{gathered}
$$

Note, following definitions and lemmas are useful in next sections. 
Definition 2.1. For a linear time-invariant operator $G: \omega \in L_{2}\left(\mathfrak{R}^{+}\right) \rightarrow z \in L_{2}\left(\mathfrak{R}^{+}\right), G$ is $L_{2}$ stable if $\omega \in L_{2}(\Re)$ implies $z \in L_{2}(\Re)$. Here, $G$ is said to have $L_{2}$ gain less than or equal to $r>0$ if and only if

$$
\int_{0}^{T}\|z(t)\|^{2} d t \leq r^{2} \int_{0}^{t}\|w(t)\|^{2} d t
$$

for all $T \in \mathfrak{R}^{+}$.

Lemma 2.2 (see [21]). For system $G:(A, B, C, D)$, the $L_{2}$ gain will be less than $\gamma>0$ if there exist a positive definite matrix $X=X^{T}>0$ such that

$$
\left[\begin{array}{ccc}
X A+A^{T} X & X B & C^{T} \\
B^{T} X & -\gamma I & D^{T} \\
C & D & -\gamma I
\end{array}\right]<0 .
$$

Definition 2.3. For a linear time-invariant operator $G: w \rightarrow z, H_{2}$ norm $G$ is defined by

$$
\|G\|_{2}^{2}=\frac{1}{2 \pi} \operatorname{trace} \int_{-\infty}^{\infty} G(j \omega) G(j \omega)^{*} d \omega
$$

Lemma 2.4 (see [21]). For stable system $G:(A, B, C)$ the $H_{2}$ performance will be less than $v$ if there exist a matrix $Z$ and a positive definite matrix $X=X^{T}>0$ such that the following LMIs are feasible:

$$
\begin{gathered}
{\left[\begin{array}{cc}
A^{T} X+X A & X B \\
B^{T} X & -I
\end{array}\right]<0} \\
{\left[\begin{array}{cc}
X & C^{T} \\
C & Z
\end{array}\right]>0} \\
\operatorname{trace}(Z)<v
\end{gathered}
$$

Lemma 2.5 (see [22]). For a positive scalar $\varepsilon^{*}$ and symmetric matrices $S_{1}, S_{2}$, and $S_{3}$ with appropriate dimensions, inequality

$$
S_{1}+\varepsilon S_{2}+\varepsilon^{2} S_{3}>0
$$

holds for all $\varepsilon \in\left(0, \varepsilon^{*}\right]$, if

$$
\begin{gathered}
S_{1} \geq 0, \\
S_{1}+\varepsilon^{*} S_{2}>0, \\
S_{1}+\varepsilon^{*} S_{2}+\varepsilon^{* 2} S_{3}>0 .
\end{gathered}
$$




\section{Main Result}

Here, we address stability, $H_{\infty}$ stability, $H_{2}$ performance, and multiobjective $H_{2} / H_{\infty}$ performance for a singularly perturbed system via dynamic output feedback control.

\subsection{Stability Problem}

Consider closed loop system (2.7) without disturbance $w(t)$ :

$$
E_{e} \dot{x}_{\mathrm{cl}}=\left[\begin{array}{cc}
A+B D_{c} C_{y} & B C_{c} \\
E_{\varepsilon}^{-1} B_{c} C_{y} & E_{\varepsilon}^{-1} A_{c}
\end{array}\right] x_{\mathrm{cl}},
$$

where $A, B, C_{y}$ were defined in (2.2). In the following theorem, we propose design procedure for obtaining controllers parameters such that the closed loop system (3.1) becomes asymptotically stable.

Theorem 3.1. Given an upperbound $\varepsilon^{*}$ for the singular perturbation $\varepsilon$, if there exist matrices $A_{k}, B_{k}, C_{k}, D_{k}, Y_{11}, Y_{12}, Y_{22}, X_{11}, X_{12}$, and $X_{22}$ satisfying the following LMIs

$$
\begin{gathered}
\Upsilon_{11}(0) \leq 0 \\
\Upsilon_{11}\left(\varepsilon^{*}\right)<0 \\
{\left[\frac{\left[\begin{array}{cc}
Y_{11} & \varepsilon^{*} Y_{12} \\
\varepsilon^{*} Y_{12}^{T} & \varepsilon^{*} Y_{22}
\end{array}\right] \mid\left[\begin{array}{cc}
I & 0 \\
0 & \varepsilon^{*} I
\end{array}\right]}{\left[\begin{array}{cc}
I & 0 \\
0 & \varepsilon^{*} I
\end{array}\right] \mid\left[\begin{array}{cc}
X_{11} & \varepsilon^{*} X_{12} \\
\varepsilon^{*} X_{12}^{T} & \varepsilon^{*} X_{22}
\end{array}\right]}\right]>0,} \\
{\left[\begin{array}{cc}
Y_{11} & I \\
I & X_{11}
\end{array}\right]>0}
\end{gathered}
$$

where

$$
\Upsilon_{11}\left(\varepsilon^{*}\right)=\left[\begin{array}{c|c}
A\left[\begin{array}{cc}
Y_{11} & \varepsilon^{*} Y_{12} \\
Y_{12}^{T} & Y_{22}
\end{array}\right]+B C_{k}+(*) & A+B D_{k} C_{y}+A_{k}^{T} \\
\hline(*) & {\left[\begin{array}{cc}
X_{11} & X_{12}^{T} \\
\varepsilon^{*} X_{12} & X_{22}
\end{array}\right] A+B_{k} C_{y}+(*)}
\end{array}\right] .
$$

Then, for any $\varepsilon \in\left(0, \varepsilon^{*}\right]$, the closed-loop singularly perturbed system (3.1) is asymptotically stable 
via dynamic output controller (2.4), also controller parameters are obtained from following equations:

$$
\begin{gathered}
D_{c}=D_{k} \\
C_{c}=\left(C_{k}-D_{c} C Q_{11}\right), \\
B_{c}=\vartheta_{\varepsilon}^{-1}\left(B_{k}-P_{11}^{T} B D_{c}\right), \\
A_{c}=\vartheta_{\varepsilon}^{-1}\left(A_{k}-\left(P_{11}^{T}\left(A+B D_{c} C_{y}\right) Q_{11}+\vartheta_{\varepsilon} B_{c} C_{y} Q_{11}+P_{11}^{T} B C_{c}\right)\right),
\end{gathered}
$$

where

$$
\begin{gathered}
P_{11}=\left[\begin{array}{cc}
X_{11} & \varepsilon X_{12} \\
X_{12}^{T} & X_{22}
\end{array}\right], \quad Q_{11}=\left[\begin{array}{cc}
Y_{11} & \varepsilon Y_{12} \\
Y_{12}^{T} & Y_{22}
\end{array}\right], \\
\vartheta_{\varepsilon}=\left[\begin{array}{cc}
I-\vartheta_{1}-\varepsilon \vartheta_{2} & -\vartheta_{3} \\
-\varepsilon \vartheta_{4} & I-\varepsilon \vartheta_{2}^{T}-\vartheta_{5}
\end{array}\right], \\
\vartheta_{1}=X_{11} Y_{11}, \quad \vartheta_{2}=X_{12} Y_{12}^{T}, \quad \vartheta_{3}=X_{11} Y_{12}+X_{12} Y_{22}, \\
\vartheta_{4}=X_{12}^{T} Y_{11}+X_{22} Y_{12}^{T}, \quad \vartheta_{5}=X_{22} Y_{22} .
\end{gathered}
$$

Proof. Choose the Lyapunov function as

$$
V(t)=x_{\mathrm{cl}}^{T}(t) E_{e} P_{\varepsilon} x_{\mathrm{cl}}(t)
$$

where

$$
\begin{aligned}
& E_{e} P_{\varepsilon}=P_{\varepsilon}^{T} E_{e}>0, \\
& P_{\varepsilon}=\left[\begin{array}{cc}
P_{11} & P_{12} \\
P_{12}^{T} E_{\varepsilon} & P_{22}
\end{array}\right] .
\end{aligned}
$$

From (3.10), it is concluded that

$$
E_{e} P_{\varepsilon}=P_{\varepsilon}^{T} E_{e} \Longrightarrow\left[\begin{array}{cc}
E_{\varepsilon} P_{11} & E_{\varepsilon} P_{12} \\
P_{12}^{T} E_{\varepsilon} & P_{22}
\end{array}\right]=\left[\begin{array}{cc}
P_{11}^{T} E_{\varepsilon} & E_{\varepsilon} P_{12} \\
P_{12}^{T} E_{\varepsilon} & P_{22}^{T}
\end{array}\right]
$$

and from (3.12), $P_{11}$ has following structure:

$$
E_{\varepsilon} P_{11}=P_{11}^{T} E_{\varepsilon} \Longrightarrow P_{11}=\left[\begin{array}{cc}
X_{11} & \varepsilon X_{12} \\
X_{12}^{T} & X_{22}
\end{array}\right]
$$


Now, define invert of $E_{e} P_{\varepsilon}$ as follow

$$
\begin{gathered}
\left(E_{e} P_{\varepsilon}\right)^{-1}=P_{\varepsilon}^{-1} E_{e}^{-1} \\
P_{\varepsilon}^{-1}=Q_{\varepsilon}=\left[\begin{array}{cc}
Q_{11} & E_{\varepsilon}^{-1} Q_{12} \\
Q_{12}^{T} & Q_{22}
\end{array}\right]
\end{gathered}
$$

and $Q_{11}$ has following structure:

$$
Q_{\varepsilon}\left(E_{e}\right)^{-1}=E_{e}^{-1} Q_{11}^{T} \Longrightarrow\left[\begin{array}{cc}
Q_{11} E_{\varepsilon}^{-1} & E_{\varepsilon}^{-1} Q_{12} \\
Q_{12}^{T} E_{\varepsilon}^{-1} & Q_{22}
\end{array}\right]=\left[\begin{array}{cc}
E_{\varepsilon}^{-1} Q_{11}^{T} & E_{\varepsilon}^{-1} Q_{12} \\
Q_{12}^{T} E_{\varepsilon}^{-1} & Q_{22}
\end{array}\right], \quad Q_{11}=\left[\begin{array}{cc}
Y_{11} & \varepsilon Y_{12} \\
Y_{12}^{T} & Y_{22}
\end{array}\right]
$$

Using the following equality:

$$
\left[\begin{array}{cc}
E_{\varepsilon} P_{11} & E_{\varepsilon} P_{12} \\
P_{12}^{T} E_{\varepsilon} & P_{22}
\end{array}\right]\left[\begin{array}{cc}
Q_{11} E_{\varepsilon}^{-1} & E_{\varepsilon}^{-1} Q_{12} \\
Q_{12}^{T} E_{\varepsilon}^{-1} & Q_{22}
\end{array}\right]=\left[\begin{array}{cc}
I & 0 \\
0 & I
\end{array}\right]
$$

we also have the constraint that

$$
E_{\varepsilon}\left(P_{11} Q_{11}+P_{12} Q_{12}^{T}\right) E_{\varepsilon}^{-1}=I \Longrightarrow P_{11} Q_{11}+P_{12} Q_{12}^{T}=I .
$$

Here, we define new matrices $\Pi_{1}$ and $\Pi_{2}$ as follows:

$$
\begin{gathered}
\Pi_{1}=\left[\begin{array}{ll}
Q_{11} & I \\
Q_{12}^{T} & 0
\end{array}\right], \\
P_{\varepsilon} \Pi_{1}=\Pi_{2}
\end{gathered}
$$

and $\Pi_{2}$ is obtained from (3.17) as follows:

$$
\left[\begin{array}{cc}
P_{11} & P_{12} \\
P_{12}^{T} E_{\varepsilon} & P_{22}
\end{array}\right]\left[\begin{array}{cc}
Q_{11} & I \\
Q_{12}^{T} & 0
\end{array}\right]=\left[\begin{array}{cc}
P_{11} Q_{11}+P_{12} Q_{12}^{T} & P_{11} \\
P_{12}^{T} E_{\varepsilon} Q_{11}+P_{22} Q_{12}^{T} & P_{12}^{T} E_{\varepsilon}
\end{array}\right], \quad \Pi_{2}=\left[\begin{array}{cc}
I & P_{11} \\
0 & P_{12}^{T} E_{\varepsilon}
\end{array}\right] .
$$

The derivative of the Lyapunov function (3.9) is

$$
\begin{gathered}
\dot{V}=\dot{x}_{\mathrm{cl}}^{T} E_{e} P_{\varepsilon} x_{\mathrm{cl}}+x_{\mathrm{cl}}^{T} E_{e} P_{\varepsilon} \dot{x}_{\mathrm{cl}} \\
\dot{V}<0 \Longrightarrow x_{\mathrm{cl}}^{T}\left(A_{\mathrm{cl}}^{T} P_{\varepsilon}+P_{\varepsilon}^{T} A_{\mathrm{cl}}\right) x_{\mathrm{cl}}<0,
\end{gathered}
$$

where $A_{\mathrm{cl}}$ is defined in (2.8). 
Mathematical Problems in Engineering

Sufficient condition to satisfy (3.21) is

$$
A_{\mathrm{cl}}^{T} P_{\varepsilon}+P_{\varepsilon}^{T} A_{\mathrm{cl}}<0
$$

Equation (3.22) will hold if and only if

$$
\Pi_{1}^{T} A_{\mathrm{cl}}^{T} P_{\mathcal{\varepsilon}} \Pi_{1}+\Pi_{1}^{T} P_{\mathcal{\varepsilon}}^{T} A_{\mathrm{cl}} \Pi_{1}<0,
$$

where $\Pi_{1}$ is defined in (3.18).

From (3.19), equation (3.23) can be rewritten as

$$
\Pi_{1}^{T} A_{\mathrm{cl}}^{T} \Pi_{2}+\Pi_{2}^{T} A_{\mathrm{cl}} \Pi_{1}<0
$$

With substituting $\Pi_{1}$ and $A_{\mathrm{cl}}$ from (3.18) and (2.8), respectively, we have

$$
\left[\begin{array}{cc}
I & 0 \\
P_{11}^{T} & E_{\varepsilon} P_{12}
\end{array}\right]\left[\begin{array}{cc}
A+B D_{c} C & B C_{c} \\
E_{\varepsilon}^{-1} B_{c} C & E_{\varepsilon}^{-1} A_{c}
\end{array}\right]\left[\begin{array}{cc}
Q_{11} & I \\
Q_{12}^{T} & 0
\end{array}\right]+(*)<0 .
$$

Now, we define the new variables:

$$
\begin{gathered}
C_{k}=D_{c} C Q_{11}+C_{c} Q_{12}^{T} \\
B_{k}=P_{11}^{T} B D_{c}+E_{\varepsilon} P_{12} E_{\varepsilon}^{-1} B_{c} \\
A_{k}=P_{11}^{T}\left(A+B D_{c} C_{y}\right) Q_{11}+E_{\varepsilon} P_{12} E_{\varepsilon}^{-1} B_{c} C_{y} Q_{11}+P_{11}^{T} B C_{c} Q_{12}^{T}+E_{\varepsilon} P_{12} E_{\varepsilon}^{-1} A_{c} Q_{12}^{T} .
\end{gathered}
$$

Then, from (3.13), (3.15), and (3.26), equation (3.25) can be rewritten as

$$
\Upsilon_{11}(\varepsilon)=\left[\begin{array}{c|c}
A\left[\begin{array}{cc}
Y_{11} & \varepsilon Y_{12} \\
Y_{12}^{T} & Y_{22}
\end{array}\right]+B C_{k}+(*) \mid & A+B D_{k} C_{y}+A_{k}^{T} \\
(*) & {\left[\begin{array}{cc}
X_{11} & X_{12}^{T} \\
\varepsilon X_{12} & X_{22}
\end{array}\right] A+B_{k} C_{y}+(*)}
\end{array}\right]<0
$$

Also, the condition (3.10) holds if and only if

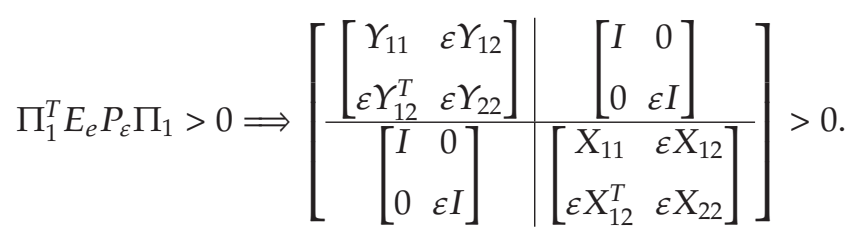

According to Lemma 2.5, the conditions (3.27) and (3.28) are valid for all $\varepsilon \in\left(0, \varepsilon^{*}\right]$, If (3.2), (3.3), (3.4), and (3.5) are satisfied. 
For computing controller parameters we obtain $P_{11}$ and $Q_{11}$ from solving LMIs in (3.2), (3.3), (3.5), and (3.6). Then from constraint (3.17) with assumption $Q_{12}=I$ we have

$$
\begin{gathered}
P_{12}=I-P_{11} Q_{11}=I-\left[\begin{array}{cc}
X_{11} & \varepsilon X_{12} \\
X_{12}^{T} & X_{22}
\end{array}\right]\left[\begin{array}{cc}
Y_{11} & \varepsilon Y_{12} \\
Y_{12}^{T} & Y_{22}
\end{array}\right]=I-\left[\begin{array}{cc}
\vartheta_{1}+\varepsilon \vartheta_{2} & \varepsilon \vartheta_{3} \\
\vartheta_{4} & \varepsilon \vartheta_{2}^{T}+\vartheta_{5}
\end{array}\right], \\
E_{\varepsilon} P_{12} E_{\varepsilon}^{-1}=\left[\begin{array}{cc}
I-\vartheta_{1}-\varepsilon \vartheta_{2} & -\vartheta_{3} \\
-\varepsilon \vartheta_{4} & I-\varepsilon \vartheta_{2}^{T}-\vartheta_{5}
\end{array}\right],
\end{gathered}
$$

where

$$
\begin{gathered}
\vartheta_{1}=X_{11} Y_{11}, \quad \vartheta_{2}=X_{12} Y_{12}^{T}, \\
\vartheta_{3}=X_{11} Y_{12}+X_{12} Y_{22}, \quad \vartheta_{4}=X_{12}^{T} Y_{11}+X_{22} Y_{12}^{T}, \\
\vartheta_{5}=X_{22} Y_{22} .
\end{gathered}
$$

Also From (3.29) and (3.26) we can obtain controller parameters from (3.7). Also from (3.7) controllers parameters are always well defined for all $\varepsilon \in\left(0, \varepsilon^{*}\right]$ and $\lim _{\varepsilon \rightarrow 0^{+}}\left[\begin{array}{ll}A_{c} & B_{c} \\ C_{c} & D_{c}\end{array}\right]$ become

$$
\begin{gathered}
D_{c 0}=D_{k} \\
C_{c 0}=\left(C_{k}-D_{c} C Q_{110}\right), \\
B_{c 0}=\vartheta_{\varepsilon 0}^{-1}\left(B_{k}-P_{110}^{T} B D_{c}\right), \\
A_{c 0}=\vartheta_{\varepsilon 0}^{-1}\left(A_{k}-\left(P_{110}^{T}\left(A+B D_{c} C_{y}\right) Q_{11}+\vartheta_{\varepsilon 0} B_{c} C_{y} Q_{110}+P_{110}^{T} B C_{c 0}\right)\right),
\end{gathered}
$$

where $\vartheta_{1}, \vartheta_{3}, \vartheta_{5}$ are defined in (3.30) and

$$
\begin{gathered}
P_{110}=\left[\begin{array}{cc}
X_{11} & 0 \\
X_{12}^{T} & X_{22}
\end{array}\right], \quad Q_{110}=\left[\begin{array}{cc}
Y_{11} & 0 \\
Y_{12}^{T} & Y_{22}
\end{array}\right], \\
\vartheta_{\varepsilon 0}=\left[\begin{array}{cc}
I-\vartheta_{1} & -\vartheta_{3} \\
0 & I-\vartheta_{5}
\end{array}\right] .
\end{gathered}
$$

This completes the proof.

Remark 3.2. Throughout the paper, it is assumed that the singular perturbation parameter $\varepsilon$ is available for feedback. Indeed, in many singular perturbation systems, the singular perturbation parameter $\varepsilon$ can be measured. In these cases, $\varepsilon$ is available for feedback, which has attracted much attention. For example, $\varepsilon$-dependent controllers were designed 
for singular perturbation systems in [22-24]. Since $\varepsilon$ is usually very small, an $\varepsilon$-dependent controller may be ill-conditioning as $\varepsilon$ tends to zero. Thus, it is a key task to ensure the obtained controller to be well defined. This problem will be discussed later.

\section{2. $H_{\infty}$ Performance}

Consider the closed loop system (2.7) with regulated output $z_{1}$. In following theorem, we proposed a procedure for obtaining controller parameters such that the closed loop singular perturbed system (2.7) with regulated output $z_{1}$ becomes asymptotically stable and guarantees the $H_{\infty}$ performance with attenuation parameter $\gamma$.

Theorem 3.3. Given an $H_{\infty}$ performance bound $\gamma$ and an upperbound $\varepsilon^{*}$ for the singular perturbation $\varepsilon$, if there exist matrices $A_{k}, B_{k}, C_{k}, D_{k}, Y_{11}, Y_{12}, Y_{22}, X_{11}, X_{12}$, and $X_{22}$ satisfying the following LMIs

$$
\begin{aligned}
& \psi_{11}(0)=\left[\begin{array}{c|c}
\Upsilon_{11}(0) & \multicolumn{2}{|c}{\Upsilon_{12}(0)} \\
\hline(*) & \begin{array}{c}
-\gamma I \\
D_{z w 1}+D_{z u 1} D_{k} D_{y w} \\
(*)
\end{array}
\end{array}\right] \leq 0, \\
& \psi_{11}\left(\varepsilon^{*}\right)=\left[\begin{array}{c|cc}
\Upsilon_{11}\left(\varepsilon^{*}\right) & \multicolumn{2}{|c}{\Upsilon_{12}\left(\varepsilon^{*}\right)} \\
\hline(*) & -\gamma I & D_{z w 1}+D_{z u 1} D_{k} D_{y w} \\
& (*) & -\gamma I
\end{array}\right]<0 \text {, }
\end{aligned}
$$

where $\Upsilon_{11}\left(\varepsilon^{*}\right)$ defined in (3.6) $\Upsilon_{12}\left(\varepsilon^{*}\right)$ is

$$
\Upsilon_{12}\left(\varepsilon^{*}\right)=\left[\begin{array}{cc}
B_{w}+B D_{k} D_{y w} & {\left[\begin{array}{c|c}
Y_{11} & Y_{12}^{T} \\
\hline \varepsilon^{*} Y_{12} & Y_{22}
\end{array}\right] C_{z}^{T}+C_{k}^{T} D_{z u 1}^{T}} \\
{\left[\begin{array}{c|c}
X_{11} & X_{12}^{T} \\
\hline \varepsilon^{*} X_{12} & X_{22}
\end{array}\right] B_{w}+B_{k} D_{y w}} & C_{z 1}^{T}+C_{y}^{T} D_{k}^{T} D_{z u 1}^{T}
\end{array}\right] .
$$

Then, for any $\varepsilon \in\left(0, \varepsilon^{*}\right]$, the closed-loop singularly perturbed system (2.7) is asymptotically stable and with an $H_{\infty}$-norm less than or equal to $\gamma$, also parameters controller are obtained from (3.7).

Proof. According to Lemma 2.2, the closed-loop singularly perturbed system (2.7) is asymptotically stable and the $L_{2}$ gain will be less or equal $\gamma$ if (3.10) and following inequality are satisfied:

$$
\left[\begin{array}{ccc}
A_{\mathrm{cl}}^{T} P_{\varepsilon}+P_{\varepsilon}^{T} A_{\mathrm{cl}} & P_{\varepsilon}^{T} B_{\mathrm{cl}} & C_{\mathrm{cl} 1}^{T} \\
(*) & -\gamma I & D_{\mathrm{cl} 1}^{T} \\
(*) & (*) & -\gamma I
\end{array}\right]<0
$$

Also $A_{\mathrm{cl}}, B_{\mathrm{cl}}, C_{\mathrm{cl} 11}$ and $D_{\mathrm{cl} 1}$ are defined in (2.8). 
By pre- and postmultiplying with matrices $\operatorname{diag}\left(\Pi_{1}, I, I\right)$ and $\operatorname{diag}\left(\Pi_{1}^{T}, I, I\right)$, respectively, it is concluded that:

$$
\left[\begin{array}{ccc}
\Pi_{1}^{T} A_{\mathrm{cl}}^{T} \Pi_{2}+\Pi_{2}^{T} A_{\mathrm{cl}} \Pi_{1} & \Pi_{2}^{T} B_{\mathrm{cl}} & \Pi_{1}^{T} C_{\mathrm{cl} 1}^{T} \\
(*) & -\gamma I & D_{\mathrm{cl} 1}^{T} \\
(*) & (*) & -\gamma I
\end{array}\right]<0,
$$

where $\Pi_{1}$ is defined in (3.18). According to proof of Theorem 3.1, $\Pi_{1}^{T} A_{\mathrm{cl}}^{T} \Pi_{2}+\Pi_{2}^{T} A_{\mathrm{cl}} \Pi_{1}^{T}$ is obtained. Also $\Pi_{2}^{T} B_{\mathrm{cl}}$ and $\Pi_{1}^{T} C_{\mathrm{cl} 1}^{T}$ are presented as follows:

$$
\begin{aligned}
\Pi_{2}^{T} B_{\mathrm{cl}}=\left[\begin{array}{cc}
I & 0 \\
P_{11}^{T} & E_{\varepsilon} P_{12}
\end{array}\right]\left[\begin{array}{c}
B_{w}+B D_{c} D_{y w} \\
E_{\varepsilon}^{-1} B_{c} D_{y w}
\end{array}\right] & =\left[\begin{array}{c}
B_{w}+B D_{k} D_{y w} \\
P_{11}^{T} B_{w}+P_{11}^{T} B D_{k} D_{y w}+E_{\varepsilon} P_{12} E_{\varepsilon}^{-1} B_{c} D_{y w}
\end{array}\right] \\
& =\left[\begin{array}{c}
B_{w}+B D_{k} D_{y w} \\
P_{11}^{T} B_{w}+B_{k} D_{y w}
\end{array}\right], \\
\Pi_{1}^{T} C_{\mathrm{cl} 1}^{T}=\left[\begin{array}{cc}
Q_{11}^{T} & Q_{12} \\
I & 0
\end{array}\right]\left[\begin{array}{c}
C_{z 1}^{T}+C_{y}^{T} D_{c}^{T} D_{z u 1}^{T} \\
C_{c}^{T} D_{z u 1}^{T}
\end{array}\right] & =\left[\begin{array}{c}
Q_{11} C_{z 1}^{T}+Q_{11} C_{y}^{T} D_{c}^{T} D_{z u 1}^{T}+Q_{12} C_{c}^{T} D_{z u 1}^{T} \\
C_{z 1}^{T}+C_{y}^{T} D_{c}^{T} D_{z u 1}^{T}
\end{array}\right] \\
& =\left[\begin{array}{c}
Q_{11} C_{z 1}^{T}+C_{k}^{T} D_{z u 1}^{T} \\
C_{z 1}^{T}+C_{y}^{T} D_{k}^{T} D_{z u 1}^{T}
\end{array}\right] .
\end{aligned}
$$

From (3.36), and (3.37), we have

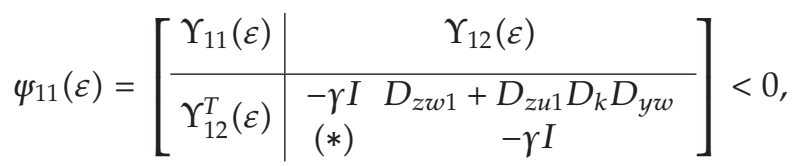

where $\Upsilon_{11}(\varepsilon)$ defined in (3.27) and

$$
\Upsilon_{12}(\varepsilon)=\left[\begin{array}{cc}
B_{w}+B D_{k} D_{y w} & {\left[\begin{array}{c|c}
Y_{11} & Y_{12}^{T} \\
\hline \varepsilon Y_{12} & Y_{22}
\end{array}\right] C_{z 1}^{T}+C_{k}^{T} D_{z u 1}^{T}} \\
{\left[\begin{array}{c|c}
X_{11} & X_{12}^{T} \\
\hline \varepsilon X_{12} & X_{22}
\end{array}\right] B_{w}+B_{k} D_{y w}} & C_{z 1}^{T}+C_{y}^{T} D_{k}^{T} D_{z u 1}^{T}
\end{array}\right] .
$$

According to Lemma 2.5, the condition (3.38) is satisfied for all $\varepsilon \in\left(0, \varepsilon^{*}\right]$, if (3.33) is satisfied. (3.4) and (3.5) compute from procedure similar to proof of Theorem 3.1 and this completes the proof.

\section{3. $\mathrm{H}_{2}$ Performance}

Consider the closed loop system (2.7) with regulated output $z_{2}$, assume $A_{\mathrm{cl}}$ is stable, $D_{z w 2}=0$ and $D_{y w}=0$. In following theorem, we proposed a procedure for obtaining controller 
parameters such that the closed loop singularly perturbed system (2.7) with regulated output $z_{2}$ guarantees the $\mathrm{H}_{2}$ performance with attenuation parameter $v$. First we propose the following lemma that is effective in proof of Theorem 3.5.

Lemma 3.4. The closed-loop singularly perturbed $G:\left(A_{\mathrm{cl}}, B_{\mathrm{cl}}, C_{\mathrm{cl}}\right)$ has the $H_{2}$ performance with attenuation parameter $v$ if following LMIs hold

$$
\begin{gathered}
{\left[\begin{array}{cc}
A_{\mathrm{cl}}^{T} P_{\varepsilon}+P_{\varepsilon}^{T} A_{\mathrm{cl}} & P_{\varepsilon}^{T} B_{\mathrm{cl}} \\
(*) & -I
\end{array}\right]<0,} \\
{\left[\begin{array}{cc}
P_{\varepsilon}^{T} E_{\varepsilon} & P_{\varepsilon}^{T} C_{\mathrm{cl}}^{T} \\
(*) & Z
\end{array}\right]>0,} \\
\operatorname{trace}(Z)<v .
\end{gathered}
$$

Proof. Consider the following closed-loop singular perturbed system:

$$
\begin{gathered}
\dot{x}(t)=E_{e}^{-1} A_{\mathrm{cl}} x(t)+E_{e}^{-1} B_{\mathrm{cl}} v(t), \\
z=C_{\mathrm{cl}} x(t),
\end{gathered}
$$

where $E_{e}$ is defined in (2.8). From Definition 2.3 we have the following equality:

$$
\|G\|_{H_{2}}^{2}=\int_{0}^{\infty} \operatorname{trace}\left(C_{\mathrm{cl}} e^{E_{e}^{-1} A_{\mathrm{cl}} t} E_{e}^{-1} B_{\mathrm{cl}} B_{\mathrm{cl}}^{T} E_{e}^{-1} e^{A_{\mathrm{cl}}^{T} E_{e}^{-1} t} C_{\mathrm{cl}}^{T}\right) d t
$$

Now we define symmetric matrix $W_{\varepsilon}$ as follows:

$$
W_{\varepsilon}=\int_{0}^{\infty}\left(e^{E_{e}^{-1} A_{\mathrm{cl}} t} E_{e}^{-1} B_{\mathrm{cl}} B_{\mathrm{cl}}^{T} E_{e}^{-1} e^{A_{\mathrm{cl}}^{T} E_{e}^{-1} t}\right) d t
$$

$\|G\|_{H_{2}}^{2}$ is obtained from the following equality:

$$
\|G\|_{H_{2}}^{2}=\operatorname{trace}\left(C_{\mathrm{cl}} W_{\varepsilon} C_{\mathrm{cl}}^{T}\right)
$$

$W_{e}$ can be obtained from the following equation:

$$
E_{e}^{-1} A_{\mathrm{cl}} W_{\varepsilon}+W_{\varepsilon} A_{\mathrm{cl}}^{T} E_{e}^{-1}+E_{e}^{-1} B_{\mathrm{cl}} B_{\mathrm{cl}}^{T} E_{e}^{-1}=0 .
$$

Suppose that there exist the matrix $X_{\varepsilon}$ with following structure:

$$
X_{\varepsilon}=\left[\begin{array}{cc}
X_{11}^{\prime} & E_{\varepsilon}^{-1} X_{12}^{\prime} \\
X_{12}^{\prime T} & X_{22}^{\prime}
\end{array}\right]
$$


that satisfies the following inequality:

$$
W_{\varepsilon}<X_{\varepsilon} E_{e}^{-1}
$$

From (3.49), equation (3.47) can be rewritten as follow:

$$
E_{e}^{-1} A_{\mathrm{cl}} X_{\varepsilon} E_{e}^{-1}+E_{e}^{-1} X_{\varepsilon}^{T} A_{\mathrm{cl}}^{T} E_{e}^{-1}+E_{e}^{-1} B_{\mathrm{cl}} B_{\mathrm{cl}}^{T} E_{e}^{-1}<0
$$

Now, pre- postmultiplying (3.50) with $E_{e}$ we have

$$
A_{\mathrm{cl}} X_{\varepsilon}+X_{\varepsilon}^{T} A_{\mathrm{cl}}^{T}+B_{\mathrm{cl}} B_{\mathrm{cl}}^{T}<0
$$

Also, from (3.46) and (3.49) we have

$$
\|G\|_{H_{2}}^{2}=\operatorname{trace}\left(C_{\mathrm{cl}} W_{\varepsilon} C_{\mathrm{cl}}^{T}\right)<\operatorname{trace}\left(C_{\mathrm{cl}} X_{\varepsilon} E_{e}^{-1} C_{\mathrm{cl}}^{T}\right)<v
$$

This is equivalent to the existence of $Z$ such that

$$
\begin{gathered}
C_{\mathrm{cl}} X_{\varepsilon} E_{e}^{-1} C_{\mathrm{cl}}^{T}<Z \\
\operatorname{trace}(Z)<v
\end{gathered}
$$

by using of Schur complement on (3.51) and (3.53) we can conclude

$$
\begin{gathered}
{\left[\begin{array}{cc}
X_{\varepsilon}^{T} A_{\mathrm{cl}}^{T}+A_{\mathrm{cl}} X_{\varepsilon} & B_{\mathrm{cl}} \\
(*) & -I
\end{array}\right]<0,} \\
{\left[\begin{array}{cc}
E_{e} P_{\varepsilon} & C_{\mathrm{cl}}^{T} \\
(*) & Z
\end{array}\right]>0}
\end{gathered}
$$

with assumption $X_{\varepsilon}^{-1}=P_{\varepsilon}$ and pre- and postmultiplying (3.54) by $\operatorname{diag}\left(P_{\varepsilon}^{T}, I\right)$ and $\operatorname{diag}\left(P_{\varepsilon}, I\right)$, respectively, we have

$$
\begin{gathered}
{\left[\begin{array}{cc}
A_{\mathrm{cl}}^{T} P_{\varepsilon}+P_{\varepsilon}^{T} A_{\mathrm{cl}} & P_{\varepsilon} B_{\mathrm{cl}} \\
(*) & -I
\end{array}\right]<0,} \\
{\left[\begin{array}{cc}
E_{\mathcal{e}} P_{\varepsilon} & P_{\varepsilon}^{T} C_{\mathrm{cl}}^{T} \\
(*) & Z
\end{array}\right]>0,} \\
\operatorname{trace}(Z)<v .
\end{gathered}
$$

This completes the proof. 
Theorem 3.5. Given an $\mathrm{H}_{2}$ performance bound $v$ and an upperbound $\varepsilon^{*}$ for the singular perturbation $\varepsilon$, if there exist matrices $A_{k}, B_{k}, C_{k}, D_{k}, Y_{11}, Y_{12}, Y_{22}, X_{11}, X_{12}$, and $X_{22}$ such that trace $(Z)<v$ andsatisfying the following LMIs:

$$
\begin{aligned}
& \tilde{\psi}_{11}(0)=\left[\begin{array}{cc}
\Upsilon_{11}(0) & \tilde{\Upsilon}_{12}(0) \\
(*) & -I
\end{array}\right] \leq 0, \\
& \tilde{\psi}_{11}\left(\varepsilon^{*}\right)=\left[\begin{array}{cc}
\Upsilon_{11}\left(\varepsilon^{*}\right) & \tilde{\Upsilon}_{12}\left(\varepsilon^{*}\right) \\
(*) & -I
\end{array}\right]<0, \\
& {\left[\begin{array}{cccc}
\left.\left[\begin{array}{cccc}
Y_{11} & 0 & I & 0 \\
0 & 0 & 0 & 0 \\
I & 0 & X_{11} & 0 \\
0 & 0 & 0 & 0
\end{array}\right] \mid \begin{array}{cc}
Y_{11} & Y_{12} \\
0 & Y_{22}
\end{array}\right] C_{z 2}^{T}+C_{k}^{T} D_{z u 2}^{T} \\
C_{z 2}^{T}+C_{y}^{T} D_{k}^{T} D_{z u 2}^{T} \\
*
\end{array}\right] \geq 0 \text {, }}
\end{aligned}
$$

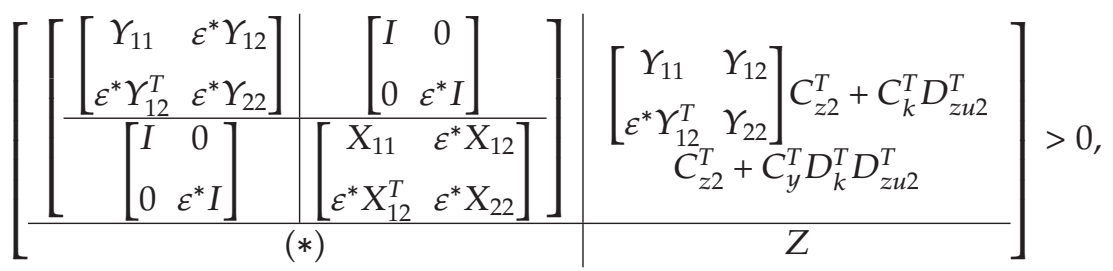

where $\Upsilon_{11}\left(\varepsilon^{*}\right)$ defined in (3.6) and $\tilde{\Upsilon}_{12}\left(\varepsilon^{*}\right)$ is

$$
\left.\tilde{\Upsilon}_{12}\left(\varepsilon^{*}\right)=\left[\begin{array}{c|c}
B_{w} \\
X_{11} & X_{12}^{T} \\
\hline \varepsilon^{*} X_{12} & X_{22}
\end{array}\right] B_{w}\right] .
$$

Then, for any $\varepsilon \in\left(0, \varepsilon^{*}\right]$, the closed-loop singularly perturbed system (2.7) is asymptotically stable and with an $\mathrm{H}_{2}$-norm less than or equal to $v$, also parameters controller are obtained from (3.7).

Proof. The proof is similar to proof of Theorem 3.3. From Lemma 3.4, the closed-loop singularly perturbed system (2.7) has $H_{2}$ performance less than or equal $v$ if (3.40), (3.41) and (3.42) are satisfied.

From (3.18) and (3.37), multiplying inequalities (3.40) and (3.41), by the matrices $\operatorname{diag}\left(\Pi_{1}, I, I\right)$ and $\operatorname{diag}\left(\Pi_{1}^{T}, I, I\right)$, gives 


$$
\begin{aligned}
& \tilde{\psi}_{11}(\varepsilon)=\left[\begin{array}{cc}
\Upsilon_{11}(\varepsilon) & \tilde{\Upsilon}_{12}(\varepsilon) \\
(*) & -I
\end{array}\right]<0,
\end{aligned}
$$

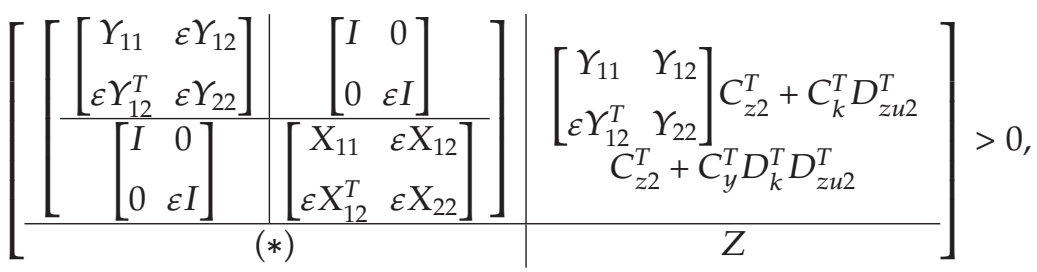

where:

$$
\tilde{\Upsilon}_{12}(\varepsilon)=\left[\begin{array}{c}
B_{w}+B D_{k} D_{y w} \\
{\left[\begin{array}{c|c}
X_{11} & X_{12}^{T} \\
\hline \varepsilon X_{12} & X_{22}
\end{array}\right] B_{w}+B_{k} D_{y w}}
\end{array}\right]
$$

According to Lemma 2.5, the inequalities (3.59) and (3.60) are valid for all $\varepsilon \in\left(0, \varepsilon^{*}\right]$, if (3.57) is satisfied and proof is complete.

\subsection{Multiobjective $H_{2} / H_{\infty}$ Performance}

Now we have got the LMIs in Theorems 3.3 and 3.5 thus we can solve the multiobjective $H_{2} / H_{\infty}$ synthesis problem easily for closed-loop singularly perturbed system (2.7) with regulated outputs $z_{1}$ and $z_{2}$.

Theorem 3.6. Given an $H_{\infty}$ performance bound $\gamma, H_{2}$ performance bound $v$ and an upperbound $\varepsilon^{*}$ for the singular perturbation $\varepsilon$, if there exist matrices $A_{k}, B_{k}, C_{k}, D_{k}, Y_{11}, Y_{12}, Y_{22}, X_{11}, X_{12}$, and $X_{22}$ such that trace $(Z)<v$ satisfying the LMIs (3.4), (3.5), (3.33), and (3.57). Then, for any $\varepsilon \in\left(0, \varepsilon^{*}\right]$, the closed-loop singularly perturbed system (2.7) is asymptotically stable and with an $\mathrm{H}_{\infty}$-norm less than or equal to $r$, an $\mathrm{H}_{2}$-norm less than or equal to $v$, also parameters controller are obtained from (3.7).

Proof. It is from the proof of Theorems 3.3 and 3.5 and omitted for save of brevity.

\section{Numerical Example}

In this section, we present a numerical results to validate the designed dynamic output feedback controller for singularly perturbed systems with $H_{\infty}$ or $H_{2}$ performance. 


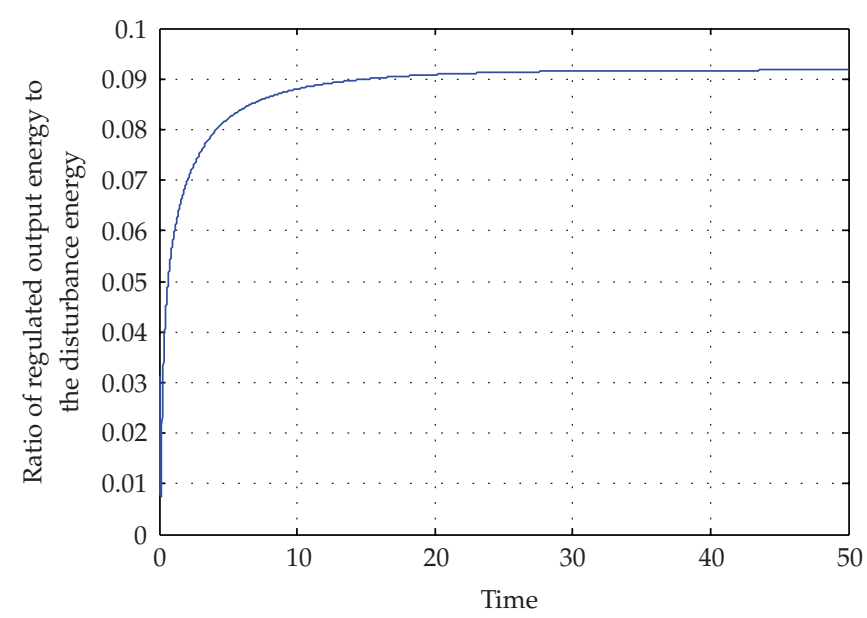

Figure 1: Simulation for full-order system (4.1) with $\varepsilon^{*}=0.1$.

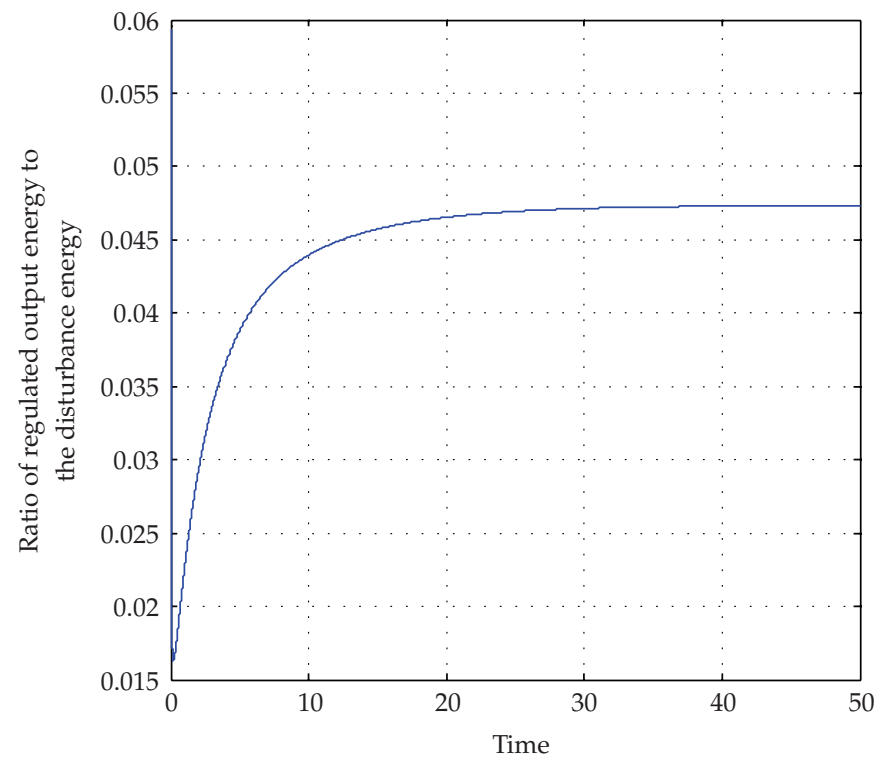

Figure 2: Simulation for full-order system (4.1) with $\varepsilon^{*}=0.001$. 


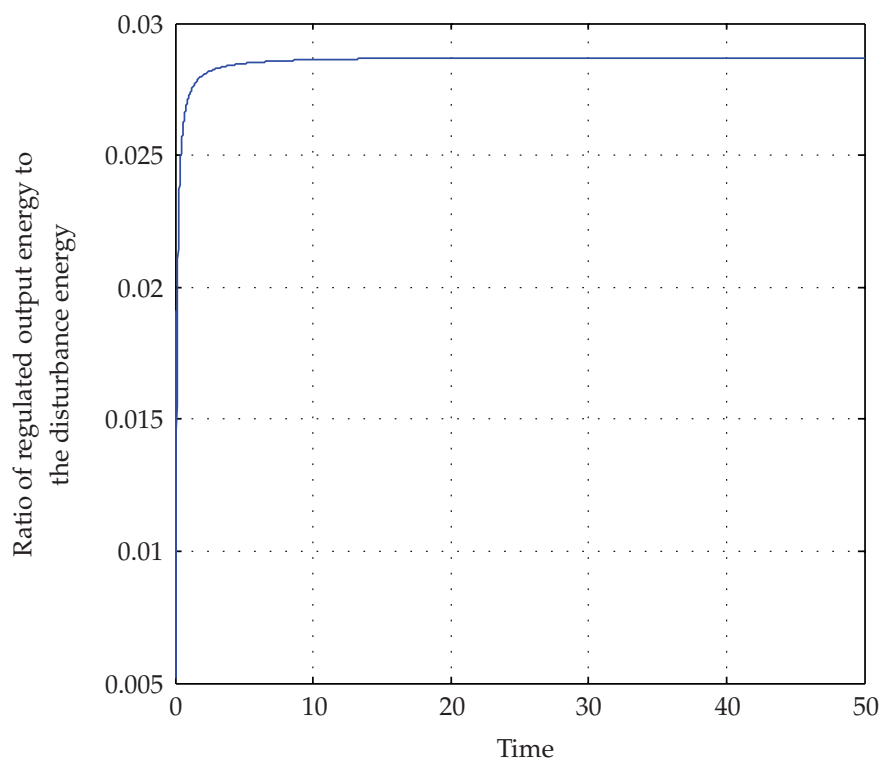

Figure 3: Simulation for reduced order system (4.3).

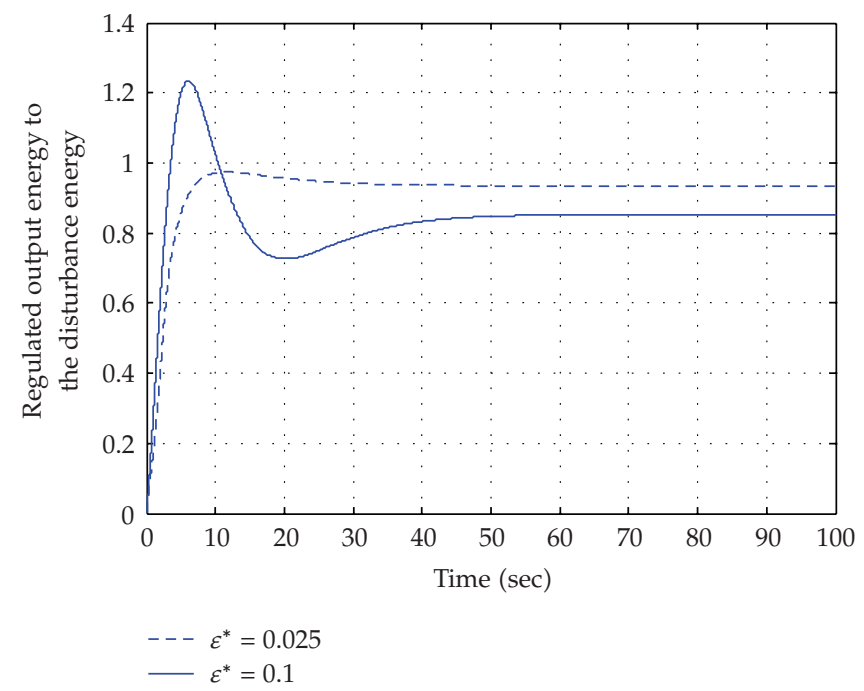

Figure 4: Simulation for full-order system (4.4) for $\varepsilon^{*}=0.1,0.025$. 


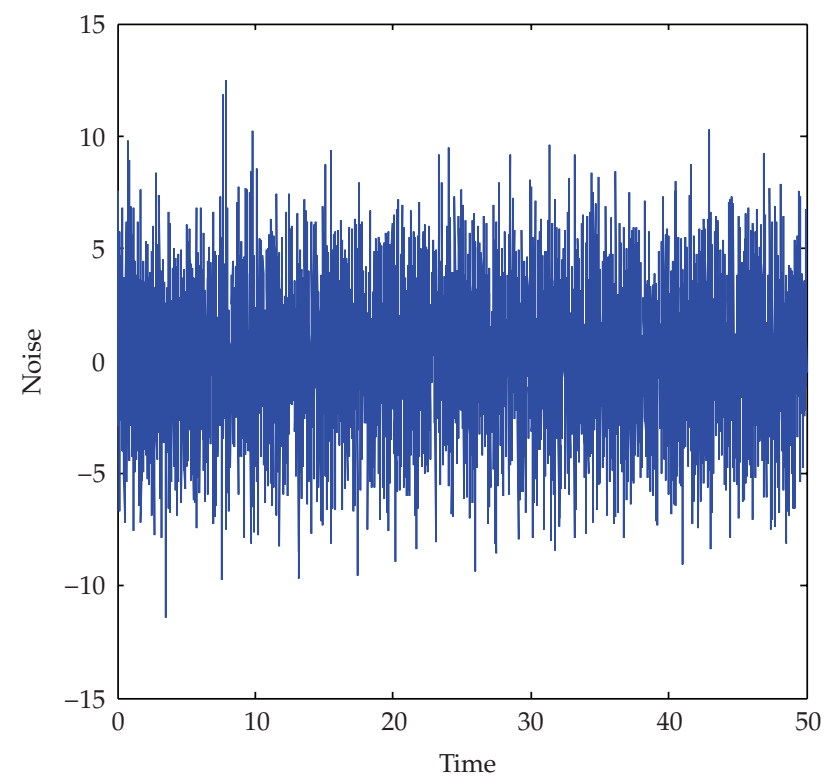

Figure 5: Band-limited white noise.

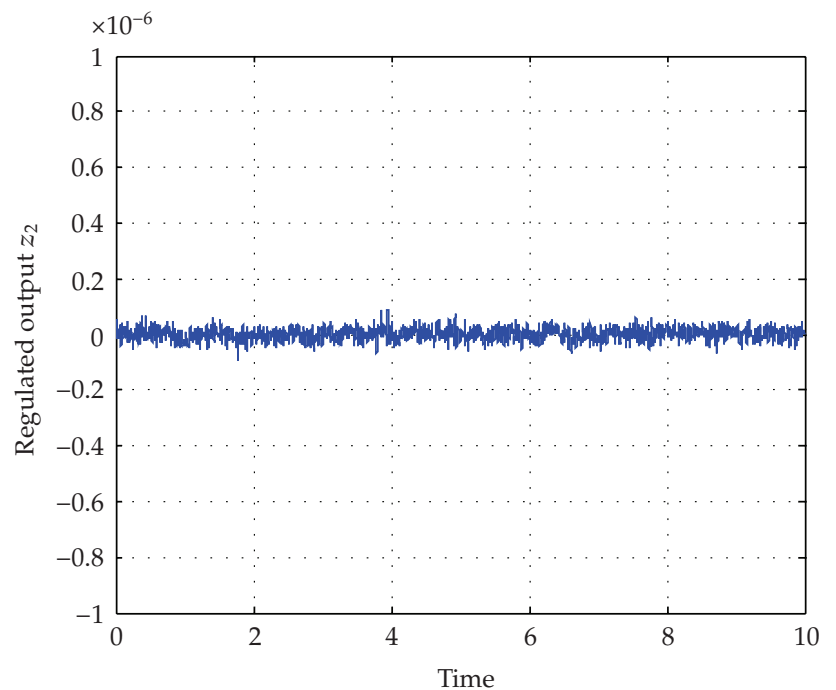

Figure 6: Regulated output $z_{2}$ for $\varepsilon^{*}=0.001$. 


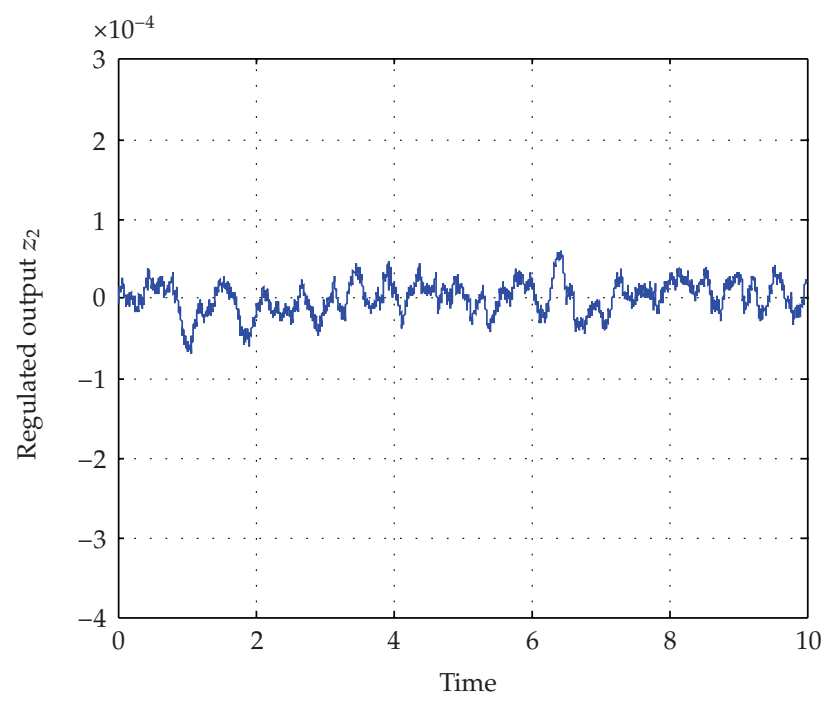

Figure 7: Regulated output $z_{2}$ for $\varepsilon^{*}=0.1$.

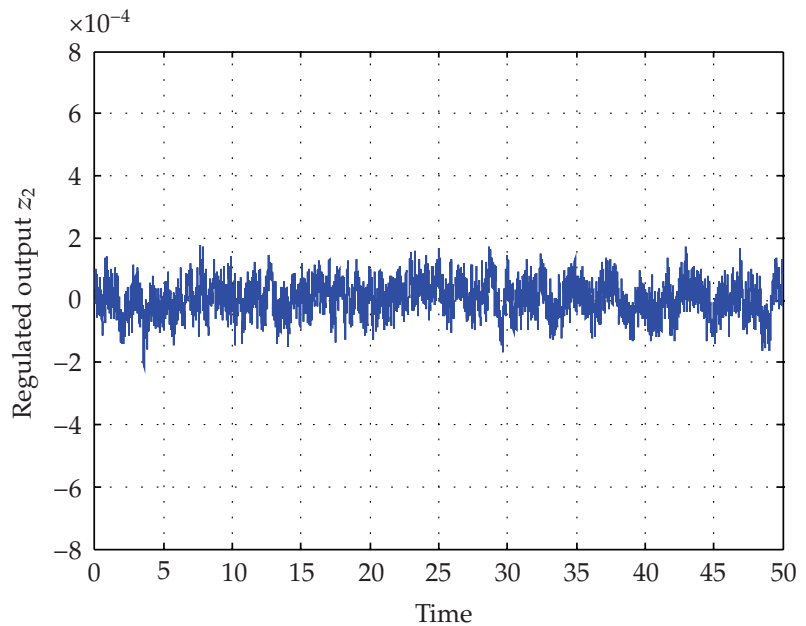

Figure 8: Regulated output $z_{2}$ for $\varepsilon^{*}=0.001$. 


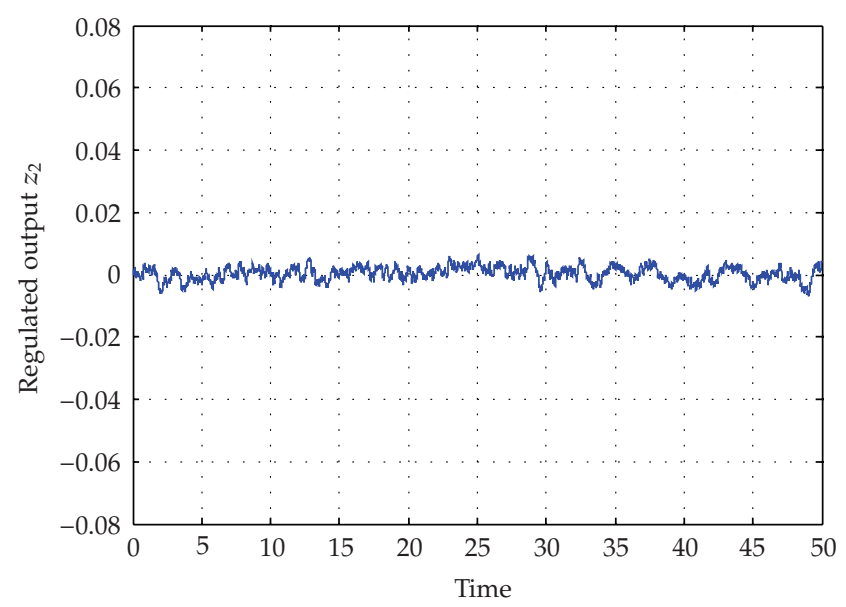

Figure 9: Regulated output $z_{2}$ for $\varepsilon^{*}=0.1$.

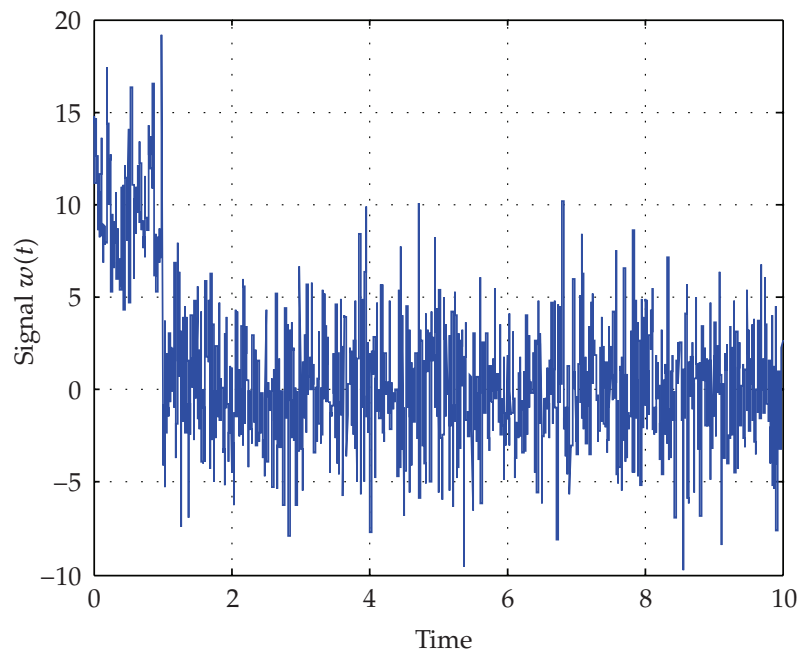

Figure 10: Disturbance signal $w(t)$.

\section{1. $H_{\infty}$ Performance}

Consider the following two-dimensional system and performance index [10]

$$
\begin{gathered}
{\left[\begin{array}{c}
\dot{x}_{1}(t) \\
\varepsilon \dot{x}_{2}(t)
\end{array}\right]=\left[\begin{array}{cc}
2 & 1 \\
-1 & -2
\end{array}\right]\left[\begin{array}{l}
x_{1} \\
x_{2}
\end{array}\right]+\left[\begin{array}{l}
2 \\
1
\end{array}\right] u(t)+\left[\begin{array}{l}
1 \\
3
\end{array}\right] w(t),} \\
z_{1}=\left[\begin{array}{ll}
2 & 0.1
\end{array}\right]\left[\begin{array}{l}
x_{1} \\
x_{2}
\end{array}\right]+0.1 w(t), \\
y(t)=\left[\begin{array}{ll}
1 & 0
\end{array}\right]\left[\begin{array}{l}
x_{1} \\
x_{2}
\end{array}\right]+w(t) .
\end{gathered}
$$




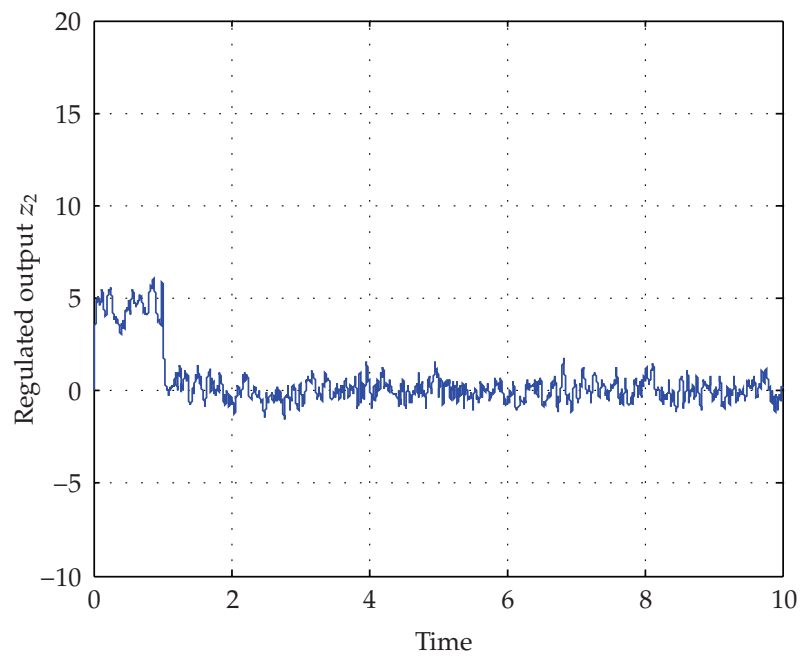

Figure 11: Regulated output $z_{2}$ with $\varepsilon^{*}=0.1$.

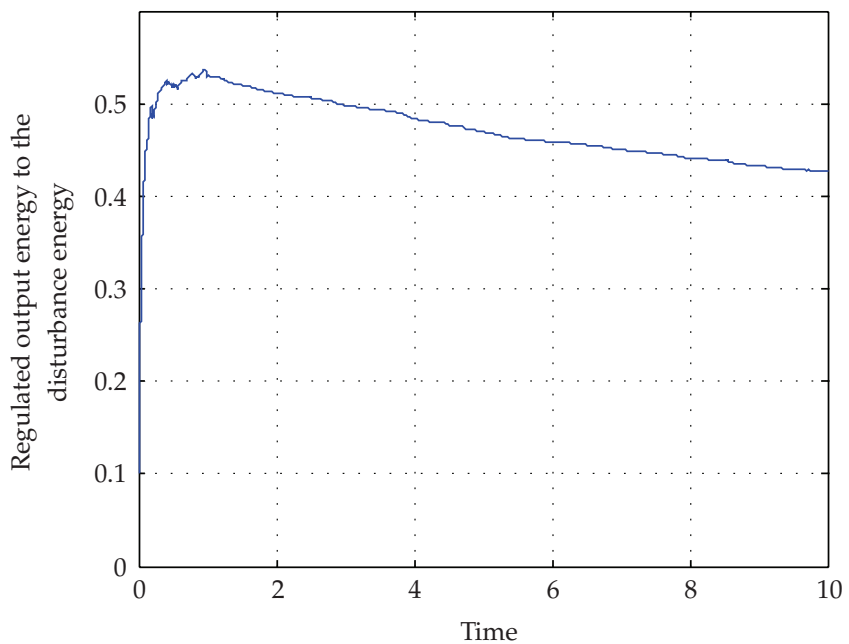

Figure 12: Simulation for $\sqrt{\int_{0}^{t} z_{1}^{T}(s) z_{1}(s) / \int_{0}^{t} w^{T}(s) w(s)}$ with $\varepsilon^{*}=0.1$. 
From Theorem 3.3, for $\varepsilon^{*}=0.1$ minimum of $\gamma$ is obtained as 0.11942 . Controller parameters are as follows:

$$
\begin{gathered}
A_{c}=\left[\begin{array}{cc}
-108.89 & -31.42 \\
91.35 & 5.201
\end{array}\right], \\
B_{c}=\left[\begin{array}{c}
-.22 \\
0.05
\end{array}\right], \\
C_{c}=\left[\begin{array}{ll}
-1793.2 & -536.1
\end{array}\right], \\
D_{c}=-4.7658 .
\end{gathered}
$$

For upperband $\varepsilon^{*}=0.001$, minimum $\gamma$ is calculated as 0.1 . As we expect $\gamma_{\varepsilon^{*}=0.001} \leq \gamma_{\varepsilon^{*}=0.1}$.

From $\varepsilon=0$, we obtain reduced order dynamic as follows:

$$
\begin{gathered}
\dot{x}_{1}(t)=1.5 x_{1}(t)+2.5 u(t)+2.5 w(t), \\
z_{1}=1.95 x_{1}(t)+0.05 u(t)+0.15 w(t), \\
y(t)=x_{1}(t)+w(t) .
\end{gathered}
$$

Here, minimum value $\gamma$ is calculated as 0.029 . For $w=e^{-0.1 t} \sin (10 t)$, Figures 1,2 , and 3 exhibit $\sqrt{\int_{0}^{t} z_{1}^{T}(s) z_{1}(s) d s / \int_{0}^{t} w^{T}(s) w(s) d s}$, respectively.

As a new example for $H_{\infty}$ performance, now consider an F-8 aircraft model [25]:

$$
\begin{gathered}
{\left[\begin{array}{c}
\dot{x}_{1} \\
\varepsilon \dot{x}_{2}
\end{array}\right]=\left(\left[\begin{array}{ll}
A_{1} & A_{2} \\
A_{3} & A_{4}
\end{array}\right]\right)\left[\begin{array}{l}
x_{1} \\
x_{2}
\end{array}\right]+\left(\left[\begin{array}{l}
B_{1} \\
B_{2}
\end{array}\right]\right) u+\left[\begin{array}{l}
B_{w 1} \\
B_{w 2}
\end{array}\right] w,} \\
z=\left[\begin{array}{ll}
C_{w 1} & C_{w 2}
\end{array}\right]\left[\begin{array}{l}
x_{1} \\
x_{2}
\end{array}\right], \\
y=\left[\begin{array}{ll}
C_{y 1} & C_{y 2}
\end{array}\right]\left[\begin{array}{l}
x_{1} \\
x_{2}
\end{array}\right]+\left[\begin{array}{l}
1 \\
1
\end{array}\right] w
\end{gathered}
$$

with 


$$
\begin{gathered}
A_{1}=\left[\begin{array}{cc}
-0.01357 & -0.0644 \\
0.06 & 0
\end{array}\right], \quad A_{2}=\left[\begin{array}{cc}
-0.003087 & 0 \\
0.040467 & 0
\end{array}\right], \\
A_{3}=\left[\begin{array}{cc}
-0.0453775 & 0 \\
0.07125 & 0
\end{array}\right], \quad A_{4}=\left[\begin{array}{cc}
-0.03055 & 0.075 \\
-0.075083 & -0.01674
\end{array}\right], \\
B_{1}=\left[\begin{array}{c}
-0.0004333 \\
0.0697
\end{array}\right], \quad B_{2}=\left[\begin{array}{c}
-0.052275 \\
0.019712
\end{array}\right] \\
B_{w 1}=\left[\begin{array}{c}
-0.463 \\
6.07
\end{array}\right], \quad B_{w 2}=\left[\begin{array}{c}
-4.5525 \\
-11.262499
\end{array}\right] \\
C_{y 1}=\left[\begin{array}{ll}
0 & 0 \\
1 & 0
\end{array}\right], \quad C_{y 2}=\left[\begin{array}{cc}
0 & 0.02 \\
0 & 0
\end{array}\right]
\end{gathered}
$$

From Theorem 3.3, for $\varepsilon^{*}=0.025$ minimum of $\gamma$ is obtained as 1.908. Controller parameters are as follows:

$$
\begin{aligned}
& A_{c}=\left[\begin{array}{cccc}
-17.0078 & 41.1022 & -62.0967 & 0.15894 \\
800.6192 & -2017.7 & 3070.05 & -5.964 \\
-1964.9 & 4954.9 & -7541 & 15.033 \\
39387 & -109682.3 & 165444.1 & -407.4100
\end{array}\right], \\
& B_{c}=\left[\begin{array}{cc}
-0.5966 & 0.5893 \\
29.39 & -28.98 \\
-72.249 & 70.9783 \\
1586.7 & -1406.2
\end{array}\right] \text {, }
\end{aligned}
$$

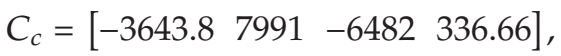

$$
\begin{aligned}
& D_{c}=\left[\begin{array}{ll}
-76.567 & -4.3010
\end{array}\right] .
\end{aligned}
$$

For upperband $\varepsilon^{*}=0.1$, minimum $\gamma$ is calculated as 3.96. As we expect $\gamma_{\varepsilon^{*}=0.025} \leq \gamma_{\varepsilon^{*}=0.1}$. For $w=e^{-0.1 t} \sin (10 t)$, Figure 4 exhibits $\sqrt{\int_{0}^{t} z_{1}^{T}(s) z_{1}(s) d s / \int_{0}^{t} w^{T}(s) w(s) d s}$. 


\section{2. $\mathrm{H}_{2}$ Performance}

Consider singular perturbed system (3.60) as

$$
\begin{gathered}
{\left[\begin{array}{c}
\dot{x}_{1}(t) \\
\varepsilon \dot{x}_{2}(t)
\end{array}\right]=\left[\begin{array}{cc}
-2 & 1 \\
-1 & -2
\end{array}\right]\left[\begin{array}{l}
x_{1}(t) \\
x_{2}(t)
\end{array}\right]+\left[\begin{array}{l}
2 \\
1
\end{array}\right] u(t)+\left[\begin{array}{l}
1 \\
3
\end{array}\right] w(t),} \\
z_{2}=\left[\begin{array}{ll}
4 & 0
\end{array}\right]\left[\begin{array}{l}
x_{1}(t) \\
x_{2}(t)
\end{array}\right]+2 u(t), \\
y(t)=\left[\begin{array}{ll}
1 & 0
\end{array}\right]\left[\begin{array}{l}
x_{1}(t) \\
x_{2}(t)
\end{array}\right] .
\end{gathered}
$$

By utilizing Theorem 3.5, minimum value of $v$ for $\varepsilon^{*}=0.1$ is obtained as 0.074 . The controller parameters are as follows:

$$
\begin{gathered}
A_{c}=\left[\begin{array}{cc}
-2558.6 & -4.289 \\
2566620 & -195.16
\end{array}\right], \\
B_{c}=\left[\begin{array}{c}
1.62 \\
-163.03
\end{array}\right], \\
C_{c}=\left[\begin{array}{ll}
-0.034 & 0.011
\end{array}\right], \\
D_{c}=-4.1
\end{gathered}
$$

For upperband $\varepsilon^{*}=0.001$, minimum $v$ is calculated as 0.0002 . As we expect $v_{\varepsilon^{*}=0.001} \leq v_{\mathcal{E}^{*}=0.1}$. Figures 5, 6, and 7 show input noise and regulated output $z_{2}$, respectively.

As a new example for $H_{2}$ performance, now consider singular perturbed system as

$$
\begin{gathered}
{\left[\begin{array}{c}
\dot{x}_{1}(t) \\
\varepsilon \dot{x}_{2}(t)
\end{array}\right]=\left[\begin{array}{cc}
-1 & 0.5 \\
1 & -2
\end{array}\right]\left[\begin{array}{l}
x_{1}(t) \\
x_{2}(t)
\end{array}\right]+\left[\begin{array}{l}
2 \\
1
\end{array}\right] u(t)+\left[\begin{array}{l}
1 \\
3
\end{array}\right] w(t),} \\
z_{2}=\left[\begin{array}{ll}
3 & 0
\end{array}\right]\left[\begin{array}{l}
x_{1}(t) \\
x_{2}(t)
\end{array}\right]+2 u(t), \\
y(t)=\left[\begin{array}{ll}
1 & 1
\end{array}\right]\left[\begin{array}{l}
x_{1}(t) \\
x_{2}(t)
\end{array}\right] .
\end{gathered}
$$

By utilizing Theorem 3.5, minimum value of $v$ for $\varepsilon^{*}=0.1$ is obtained as 0.003 . The controller parameters are as follows: 


$$
\begin{gathered}
A_{c}=\left[\begin{array}{cc}
5243.9 & -14292.8 \\
29566.58 & -80500.28
\end{array}\right], \\
B_{c}=\left[\begin{array}{c}
-0.167 \\
-9.4145
\end{array}\right], \\
C_{c}=[-77312.66829 .2], \\
D_{c}=-3.8 \times 10^{-9} .
\end{gathered}
$$

For upperband $\varepsilon^{*}=0.001$, minimum $v$ is calculated as 0.0004 . As we expect $v_{\varepsilon^{*}=0.001} \leq v_{\varepsilon^{*}=0.1}$. Input noise is shown in Figures 5, 8, and 9 show the regulated output $z_{2}$, respectively.

\section{3. $H_{\infty} / H_{2}$ Performance}

Now, consider singular perturbed system (3.60) as

$$
\begin{gathered}
{\left[\begin{array}{c}
\dot{x}_{1} \\
\varepsilon \dot{x}_{2}
\end{array}\right]=\left[\begin{array}{cc}
2 & 1 \\
-1 & -2
\end{array}\right]\left[\begin{array}{l}
x_{1} \\
x_{2}
\end{array}\right]+\left[\begin{array}{l}
2 \\
1
\end{array}\right] u+\left[\begin{array}{l}
1 \\
3
\end{array}\right] w,} \\
z_{1}=\left[\begin{array}{ll}
2 & 0.1
\end{array}\right]\left[\begin{array}{l}
x_{1} \\
x_{2}
\end{array}\right]+0.1 w \\
z_{2}=\left[\begin{array}{ll}
4 & 0
\end{array}\right]\left[\begin{array}{l}
x_{1} \\
x_{2}
\end{array}\right]+2 u, \\
y=\left[\begin{array}{ll}
1 & 0
\end{array}\right]\left[\begin{array}{l}
x_{1} \\
x_{2}
\end{array}\right]+w .
\end{gathered}
$$

According to Theorem 3.6, due to minimization of $v+\gamma$ for $\varepsilon^{*}=0.1$, the values of $v=0.14$ and $\gamma=0.233$ are calculated. By similar calculation, due to minimization of $\nu+\gamma$ for $\varepsilon^{*}=0.001$, the values of $v$ and $\gamma$ are calculated as 0.088 and 0.2018 , respectively.

Here, controller parameters are as the follows when $\varepsilon^{*}=0.1$ :

$$
\begin{gathered}
A_{c}=\left[\begin{array}{cc}
-7.4 & -9.17 \\
817.41 & -0.5
\end{array}\right], \\
B_{c}=\left[\begin{array}{c}
4.16 \\
-52.18
\end{array}\right], \\
C_{c}=\left[\begin{array}{ll}
-0.15 \times 10^{-4} & 0
\end{array}\right], \\
D_{c}=-3.206 .
\end{gathered}
$$


Figures 10 and 11 show input signal $w(t)$ and regulated output $z_{2}$. Based on the $H_{\infty} / H_{2}$, considered controller is designed to minimize effect of signal $w$ on regulated output $z_{2}$. Also the ratio of the regulated output energy to the disturbance energy is shown in Figure 12.

\section{Conclusions}

In this paper, we addressed robust $H_{2}$ and $H_{\infty}$ control via dynamic output feedback control for continuous-time singularly perturbed systems. By formulating all objectives in terms of a common Lyapunov function, the controller was designed through solving a set of inequalities. A dynamic output feedback controller was developed such that first, the $H_{\infty}$ and $\mathrm{H}_{2}$ performances of the resulting closed-loop system is less than or equal to some prescribed values, and furthermore, these performances are satisfied for all $\varepsilon \in\left(0, \varepsilon^{*}\right]$. Apart from our main results, Theorems 3.1 to 3.6 show that the $\varepsilon$-dependent controller is well defined for all $\varepsilon \in\left(0, \varepsilon^{*}\right]$ and can be reduced to an $\varepsilon$-independent providing $\varepsilon$ is sufficiently small. Finally, numerical simulations were provided to verify the proposed controller.

\section{References}

[1] P. V. Kokotović, H. K. Khalil, and J. O'Reilly, Singular Perturbation Methods in Control: Analysis and Design, Academic Press, London, UK, 1986.

[2] T. H. S. Li, M. S. Wang, and Y. Y. Sun, "Robust dynamic output feedback sliding-mode control of singular perturbation systems," JSME International Journal, Series C, vol. 38, no. 4, pp. 719-726, 1995.

[3] D.S. Naidu, "Singular perturbations and time scales in control theory and applications: an overview," Dynamics of Continuous, Discrete E Impulsive Systems. Series B, vol. 9, no. 2, pp. 233-278, 2002.

[4] S. Sen and K. B. Datta, "Stability bounds of singularity perturbed systems," IEEE Transactions on Automatic Control, vol. 38, no. 2, pp. 302-304, 1993.

[5] B.-S. Chen and C. L. Lin, "On the stability bounds of singularly perturbed systems," IEEE Transactions on Automatic Control, vol. 35, no. 11, pp. 1265-1270, 1990.

[6] G. Garcia, J. Daafouz, and J. Bernussou, "A LMI solution in the H2 optimal problem for singularly perturbed systems," in Proceedings of the American Control Conference, pp. 550-554, Philadelphia, Pa, USA, 1998.

[7] H. K. Khalil, "Output feedback control of linear two-time-scale systems," IEEE Transactions on Automatic Control, vol. 32, no. 9, pp. 784-792, 1987.

[8] V. Drăgan, P. Shi, and E. Boukas, "Control of singularly perturbed systems with Markovian jump parameters: an $H_{\infty}$ approach," Automatica, vol. 35, no. 8, pp. 1369-1378, 1999.

[9] E. Fridman, "Near-optimal $H_{\infty}$ control of linear singularly perturbed systems," IEEE Transactions on Automatic Control, vol. 41, no. 2, pp. 236-240, 1996.

[10] Z. Pan and T. Basar, " $H_{\infty}$-optimal control for singularly perturbed systems. Part I: perfect state measurements," Automatica, vol. 29, no. 2, pp. 401-423, 1993.

[11] P. Shi and V. Dragan, "Asymptotic $H_{\infty}$ control of singularly perturbed systems with parametric uncertainties," IEEE Transactions on Automatic Control, vol. 44, no. 9, pp. 1738-1742, 1999.

[12] Z. Pan and T. Başar, " $H_{\infty}$-optimal control for singularly perturbed systems. II. Imperfect state measurements," IEEE Transactions on Automatic Control, vol. 39, no. 2, pp. 280-299, 1994.

[13] J. Dong and G.-H. Yang, " $H_{\infty}$ control for fast sampling discrete-time singularly perturbed systems," Automatica, vol. 44, no. 5, pp. 1385-1393, 2008.

[14] S. Xu and G. Feng, "New results on $H_{\infty}$ control of discrete singularly perturbed systems," Automatica, vol. 45, no. 10, pp. 2339-2343, 2009.

[15] G. I. Bara and M. Boutayeb, "Static output feedback stabilization with $H_{\infty}$ performance for linear discrete-time systems," IEEE Transactions on Automatic Control, vol. 50, no. 2, pp. 250-254, 2005.

[16] Y. Xia, J. Zhang, and E. Boukas, "Control of discrete singular hybrid systems," Automatica, vol. 44, no. 10, pp. 2635-2641, 2008. 
[17] F. Long, S. Fei, Z. Fu, S. Zheng, and W. Wei, " $H_{\infty}$ control and quadratic stabilization of switched linear systems with linear fractional uncertainties via output feedback," Nonlinear Analysis: Hybrid Systems, vol. 2 , no. 1, pp. 18-27, 2008.

[18] E. Prempain and I. Postlethwaite, "Static output feedback stabilisation with $H_{\infty}$ performance for a class of plants," Systems E Control Letters, vol. 43, no. 3, pp. 159-166, 2001.

[19] D. W. C. Ho and G. Lu, "Robust stabilization for a class of discrete-time non-linear systems via output feedback: the unified LMI approach," International Journal of Control, vol. 76, no. 2, pp. 105-115, 2003.

[20] I. Masubuchi, "Output feedback controller synthesis for descriptor systems satisfying closed-loop dissipativity," Automatica, vol. 43, no. 2, pp. 339-345, 2007.

[21] C. Scherer, P. Gahinet, and M. Chilali, "Multiobjective output-feedback control via LMI optimization," IEEE Transactions on Automatic Control, vol. 42, no. 7, pp. 896-910, 1997.

[22] C. Yang and Q. Zhang, "Multiobjective control for T-S fuzzy singularly perturbed systems," IEEE Transactions on Fuzzy Systems, vol. 17, no. 1, pp. 104-115, 2009.

[23] W. Assawinchaichote, S. K. Nguang, and P. Shi, " $H_{\infty}$ output feedback control design for uncertain fuzzy singularly perturbed systems: an LMI approach," Automatica, vol. 40, no. 12, pp. 2147-2152, 2004.

[24] W. Assawinchaichote and S. K. Nguang, "Fuzzy $H_{\infty}$ output feedback control design for singularly perturbed systems with pole placement constraints: an LMI approach," IEEE Transactions on Fuzzy Systems, vol. 14, no. 3, pp. 361-371, 2006.

[25] H. Khalil and Z. Gajic, "Near-optimum regulators for stochastic linear singularly perturbed systems," IEEE Transactions on Automatic Control, vol. 29, no. 6, pp. 531-541, 1984. 


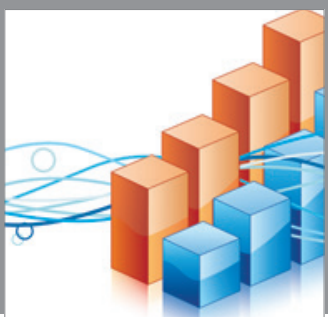

Advances in

Operations Research

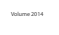

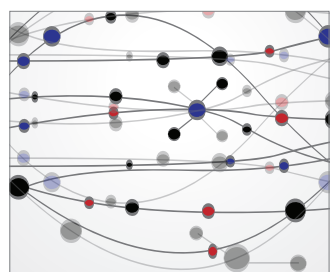

\section{The Scientific} World Journal
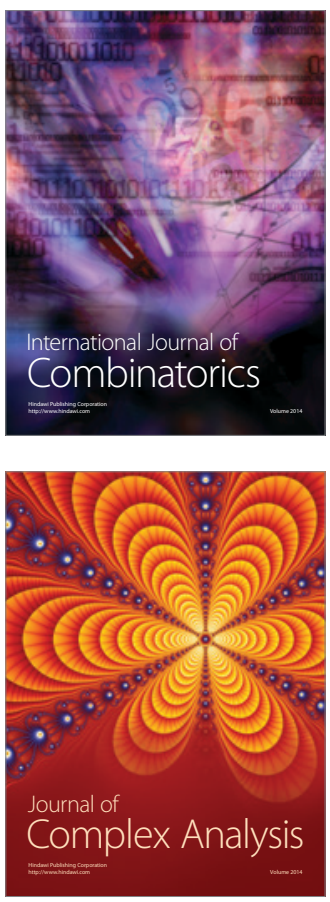

International Journal of

Mathematics and

Mathematical

Sciences
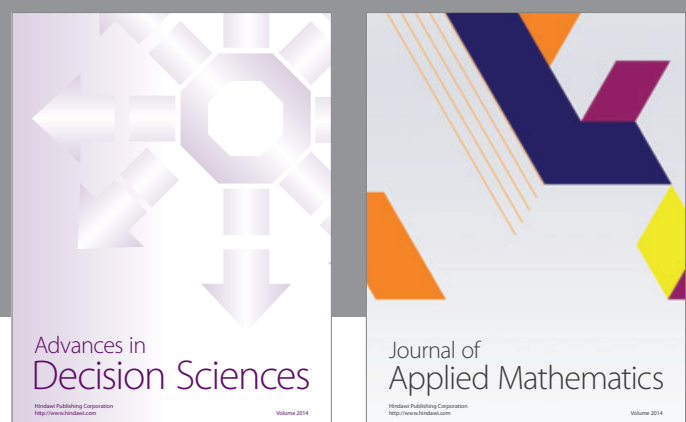

Journal of

Applied Mathematics
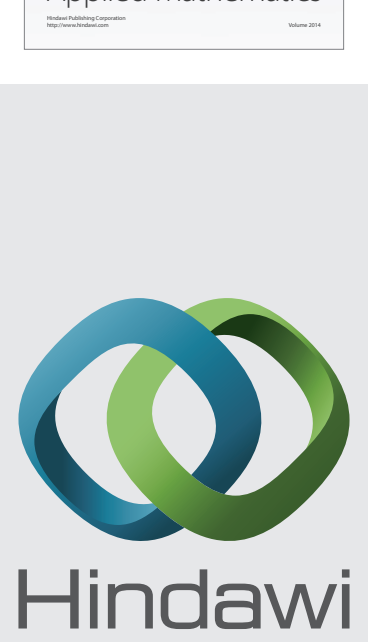

Submit your manuscripts at http://www.hindawi.com
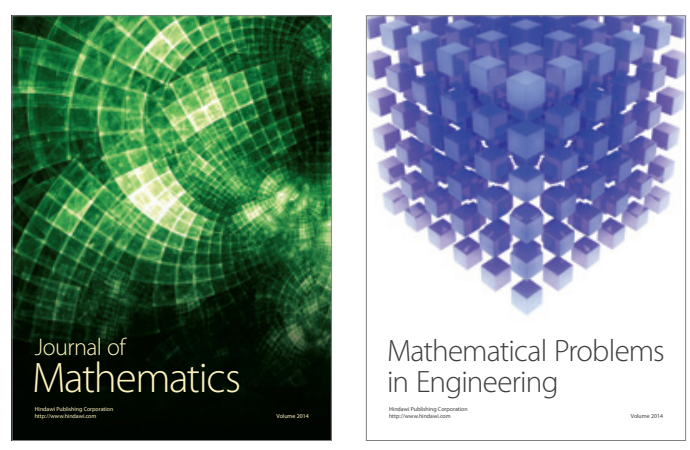

Mathematical Problems in Engineering
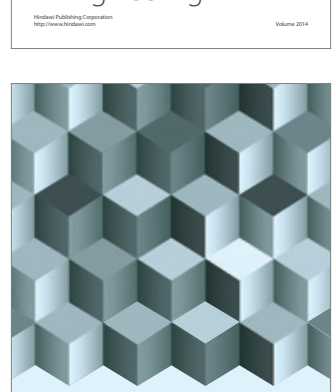

Journal of

Function Spaces
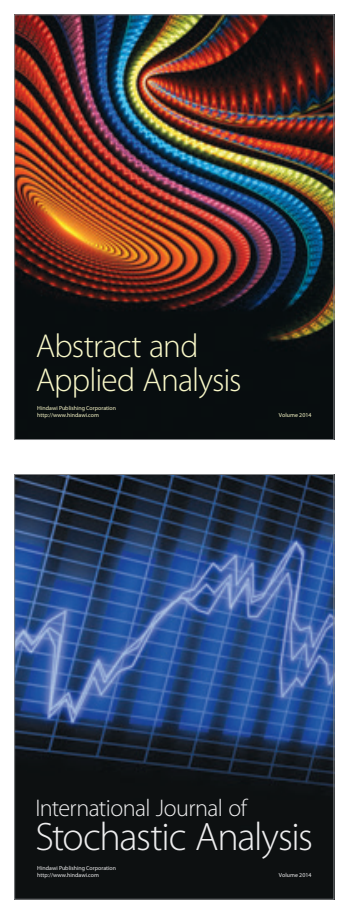

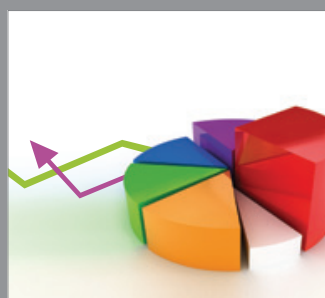

ournal of

Probability and Statistics

Promensencen
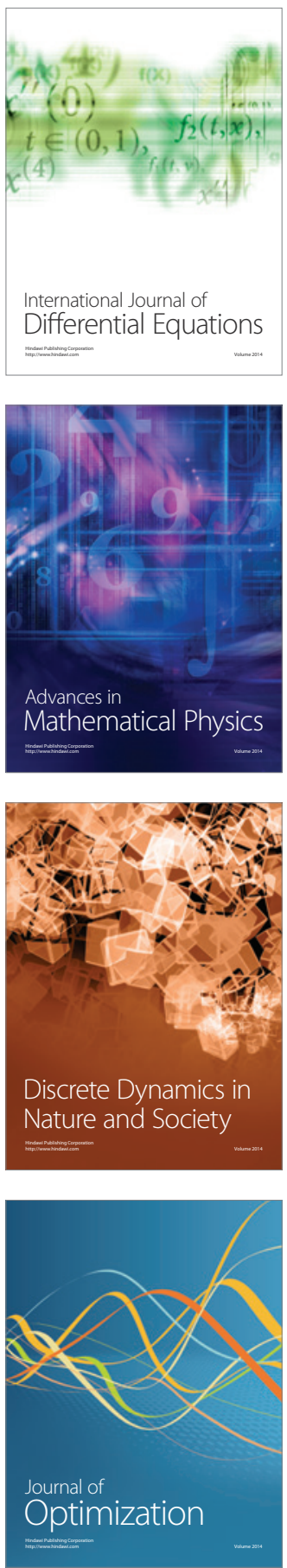